Comment

The relatively low response rate (25 per cent) and the skewed distribution of responses, with the predominance of teaching hospital trainees, casts doubt as to how representative the respondents are of trainees in the Southern Division. In addition, the majority of the respondents were interested in and engaged in research. Whilst reassuring in one way, it tells us nothing about the majority of trainees, who did not reply. One can only speculate, but the respondents may be a selfselected group interested in research, and therefore motivated to reply to the questionnaire in order to assist the 'research' of others.

Previous articles ${ }^{1.2}$ have drawn attention to the importance of adequate constructive help for trainees new to research. There is a suggestion that this study supports this view with the majority of junior trainees reporting inadequate supervision and almost a quarter unable to arrange it. It was also of note that junior trainees were perhaps less able to determine the availability and adequacy of research facilities, as they rated these as less satisfactory than did senior trainees. This may also reflect the relative inadequacy of supervision and guidance for their stage of development.

Although most trainees were based in teaching hospitals, there was, no evidence that placement in one conferred an advantage, as amongst junior trainees there was no great difference between the proportions involved in research.

In summary, the finding that the majority of the trainees who replied were involved in research is superficially reassuring but leads to questions about the poor response rate and whether this is related to lack of interest or involvement in research by the majority of trainees in the Division. The perceived lack of 'adequate' supervision by junior trainees may reflect a situation in which the supervision offered is inappropriate for the needs of the trainees.

J. A. Hollyman

M. T. Abou-Saleh

St George's Hospital Medical School

London SWI7

REFERENCES

ISZMUKLER, G. (1981) Tinker, trainer, savoir-faire: The trainee and research. Bulletin of the Royal College of Psychiatrists, 5, 47-48.

2GolDBERG, D. (1981) Encouraging psychiatric trainees to undertake research. Bulletin of the Royal College of Psychiatrists, 5, 87-88.

\section{The validity of the Membership Examination}

\section{DEAR SIRS}

Following the recent results of the Membership Examination, I hope that the newly elected members of the Collegiate Trainees' Committee will look first at this method of testing those they represent.

The point is not that it was a fundamental error to model the examination on the MRCP, which has so often seemed to reward robust self-confidence (justified or otherwise), familiarity with the obscure and simple luck. The point is not that most postgraduate exams confuse a high rate of failure with a high rate of discrimination, although many will doubt the reliability of a test which had previously failed this year's Laughlin Prize winner and which has now failed the candidate who has just won the Gaskell Medal. Nor is it that the use of videotaping should have before now led to the abandonment of the anachronistic reliance on live (and therefore varied and unpredictable) interviews. And it is not that suitability for higher clinical training cannot be assessed in circumstances as removed from normal clinical practice.

The point is simply that all these things are apparent to the most naive observer of human behaviour and should have been clear to psychiatrists long ago.

LOUIS APPLEBY

\section{Maudsley Hospital}

Denmark Hill, London SES

\section{DEAR SIRS}

In the course of a teaching session during a Journal Club Meeting with trainees, I had a very interesting and controversial argument about the need for taking an ECG as a requirement for investigations for lithium therapy and the frequency of monitoring of serum lithium levels for a stabilized patient on lithium therapy.

The trainees gave different answers, quoting from different authoritative textbooks. Now, as the multiple choice questions require specific answers and the marking system takes account of these specific answers only, it is crucial for the trainee to answer correctly if he or she is to obtain positive marks. As we all know, the state of knowledge in the field of psychiatry is still in flux and there are as many possible answers as there are questions, so the validity of the multiple choice question becomes questionable and consequently that of the examination. Naturally, the loser in such a situation is the trainee who is taking such an examination.

Perhaps the Examining Body of the College ought to review seriously the 'multiple choice question' type of examination and make appropriate amendments in the written part of the examinations.

T. HARI SINGH

Hensol Hospital,

Pontyclun, Mid-Glamorgan

\section{Deinstitutionalization in the USA}

DeAR SIRS

Dr Fagin is surely right to draw our attention to the lessons that we in Britain may learn from the experience of the deinstitutionalization movement (bandwagon?) in the United States (Bulletin, June 1985, 9, 112-114). It should not be forgotten that in England and Wales at least there has been a very substantial run-down in the in-patient psychiatric population, albeit less precipitate than that which has occurred in America.

Several additional points emerge from the American literature that may be useful as we contemplate the large scale closure of mental hospitals and their replacement by networks of 'community-based' services. Firstly, mental hospitals serve the needs of the most severely handicapped patients in ways 